\title{
A Minimal Invasive Prosthetic Solution for Small, Atrophic Edentulous Arches Using Narrow Diameter Implant and Magnetic Overdenture: A Case Report
}

\author{
Ki Young Nam* \\ Department of Dentistry, College of Medicine, Keimyung University, 700-712, Daegu, South Korea \\ *Corresponding author: nkyp@dsmc.or.kr
}

Received February 01, 2015; Revised February 23, 2015; Accepted February 27, 2015

\begin{abstract}
The rehabilitation of the fully edentulous patients could be compromised due to their diversity in terms of clinical, medical, anatomical and economic situations. Typically, narrow and atrophic edentulous ridges complicate retention, support and stability. Overdenture using attachment or implants could help the improvement of denture function and recommended due to its relative simplicity, predictability and affordability. In this report, a short stature patient under medically and physically handicapped was treated as minimal invasive prosthetic intervention using single magnetic attachment (Magfit ${ }^{\circledR}$ ) on the maxilla and 2 freestanding mini-implants (LODI ${ }^{\circledR}$ ) placed in mandible following recommendations from the 2002 McGill. For 12 months following up, patient is satisfied with the final results in functional aspects and esthetic.
\end{abstract}

Keywords: atrophic dental arch, edentulous, overdenture, narrow diameter implant, magnetic attachment

Cite This Article: Ki Young Nam, "A Minimal Invasive Prosthetic Solution for Small, Atrophic Edentulous Arches Using Narrow Diameter Implant and Magnetic Overdenture: A Case Report.” International Journal of Dental Sciences and Research, vol. 3, no. 2 (2015): 32-34. doi: 10.12691/ijdsr-3-2-3.

\section{Introduction}

Edentulous patients are a diverse group comprised of those who are hereditary, medically compromised, economically depressed and geriatric affected. Complete maxillary and mandibular dentures have been the traditional standard of therapy for more than a century, but in various clinical situations, the providing adequate support, retention and stability are still challenged though the prosthodontic skills are applied to these principles strictly toward patients. Moreover, the severely narrow and constricted dental arches commonly represent significant treatment dilemma with the factors of support, retention and stability. Generally, patients would subjectively perceive the treatment success in terms of increased prosthesis retention and stability because those factors are directly related with the chewing and speaking ability [1]. With the developments in dental implant technology, the provision of implants remains the treatment of choice meeting the functional expectations in cases where conventional prosthetic measures have failed, The most common implant treatment of choice for an edentulous jaw involved the placement of 6-8 implants or more, all fixed implant supported prosthesis [2]. However, the patients who are under anatomical, medical and financial restrictions, the treatment option should be modified into the conservative or lesser invasive one. This paper reports minimally invasive overdenture try-on to a handicapped patient with atrophic jaws supported by two narrow implant $\left(\mathrm{LODI}^{\circledR}\right.$; Locator overdenture implant system) on the anterior mandible and single magnet $\left(\right.$ Magfit $^{\circledR}$ ) attachment on maxillay cuspid.

\section{Case Report}

A 34-year-old male was referred due to his teeth absence. The patient's main complaint was the exclusion of his old denture wearing because he had been aware of its inconvenience and complications due to the lack of retention and stability. On physical and dental examinations, patient revealed an edentulous jaw state except \#13 which showed no symptom and mobility (Figure 1). Typically, his entire dimension of maxilla were shown as much smaller in size as compared with the average example and the palatal vault was flat with shallow posterior buccal sulcus extensions in prediagnostic gypsum model (Figure 2). In mandible, posterior ridge was highly resorbed enough to be shown as knife edge (Figure 3). Medical history exhibited he has been in diabetes from juvenile and on anticoagulant (aspirin) therapy due to heart prosthetic valve, in addition, he was handicapped with blindness, short stature as 147 $\mathrm{cm}$ in height. The patient was explained that he may be required a complex oral rehabilitation for fixed implant supported bridge with bone graft surgery to solve his chief complaint, eventually, he strongly expressed a desire of the minimal and effective dental treatment for the reasons of financial and general health status. In consideration of his expectation for improved new denture functions and 
the need for an economically-feasible treatment option, prosthodontic plan was made by the overdenture with minimal supportive attachments; a root supported magnetic cap (\# 13) in maxilla and an implant supported overdenture in lower part respectively.

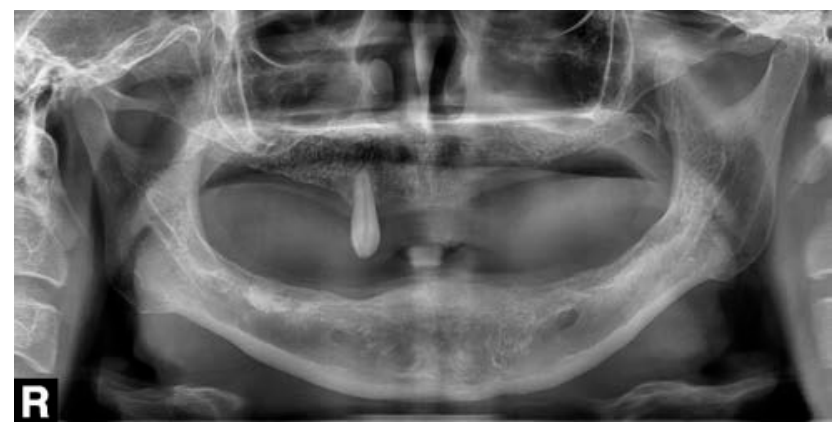

Figure 1. Pre-prosthodontic panoramic view, fully edentulous jaw except \#13 is shown with generally resorbed aspect

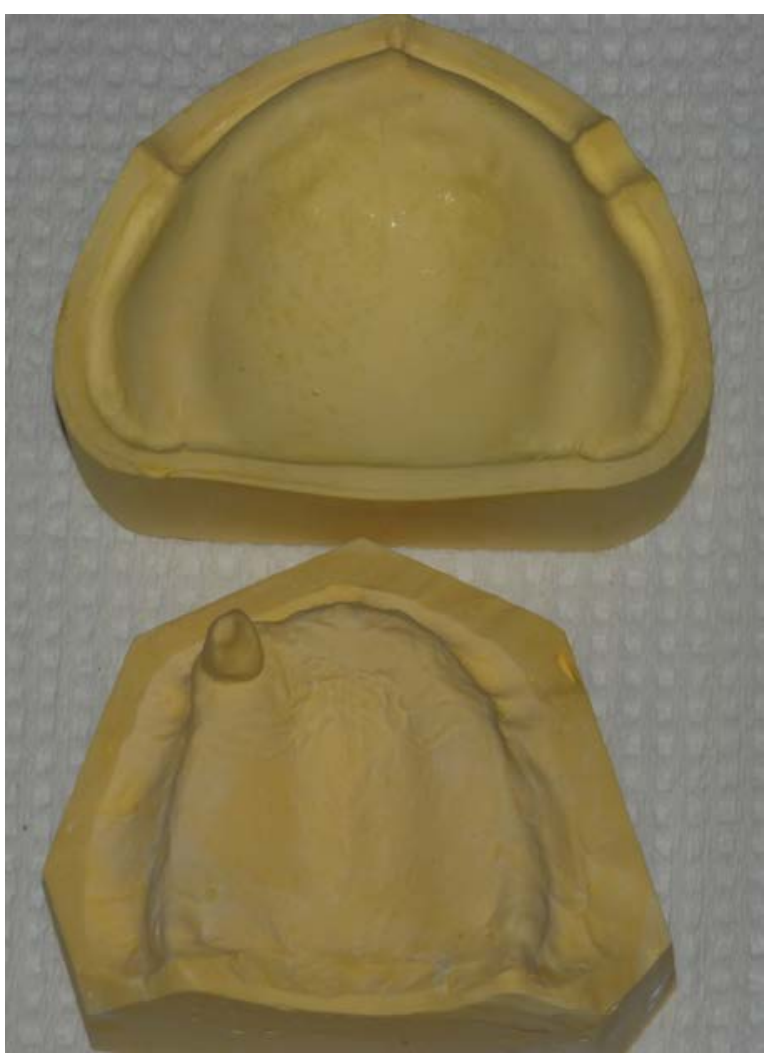

Figure 2. Constricted maxilla arch dimension (below) as compared to average model (above)

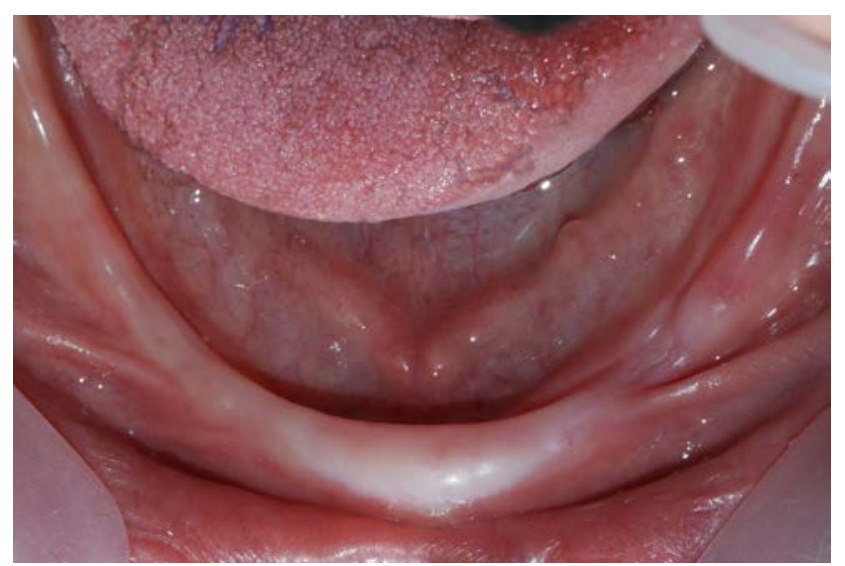

Figure 3. Narrow and severe atrophic mandible ridge

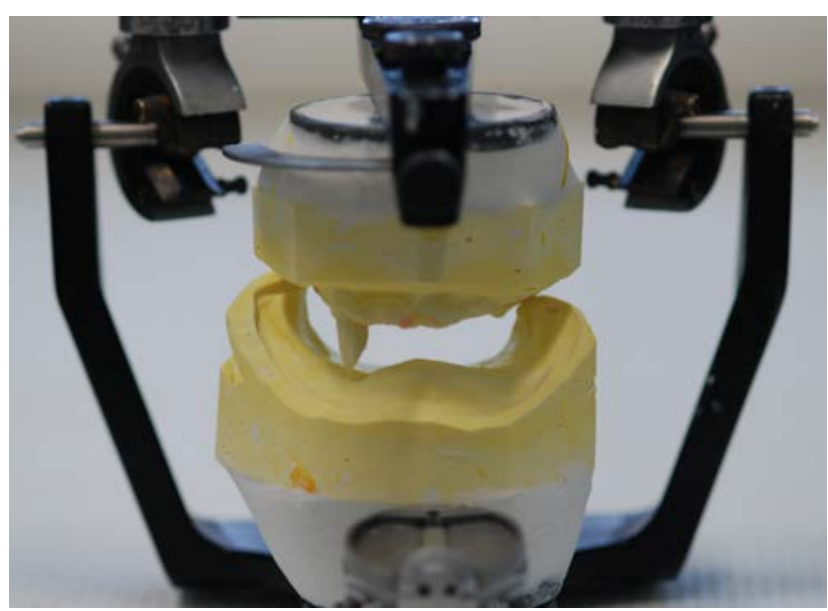

Figure 4. Transfer of intermaxillary relations of occlusion, anterior interridge space was revealed as $16 \mathrm{~mm}$.

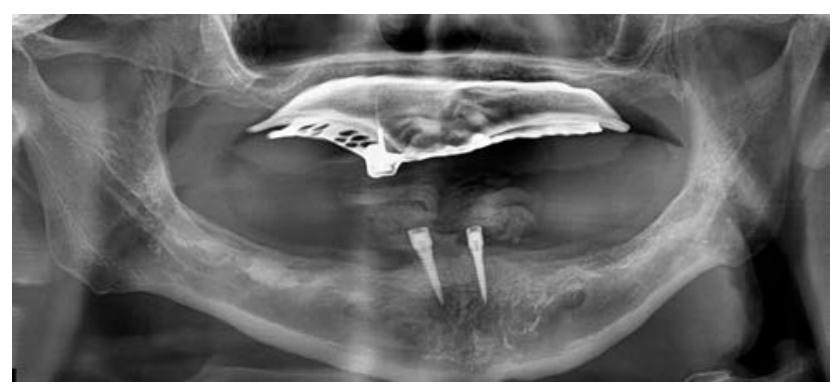

Figure 5. Two LODI installed showed apparent osseointegration after 3 month-healing periods

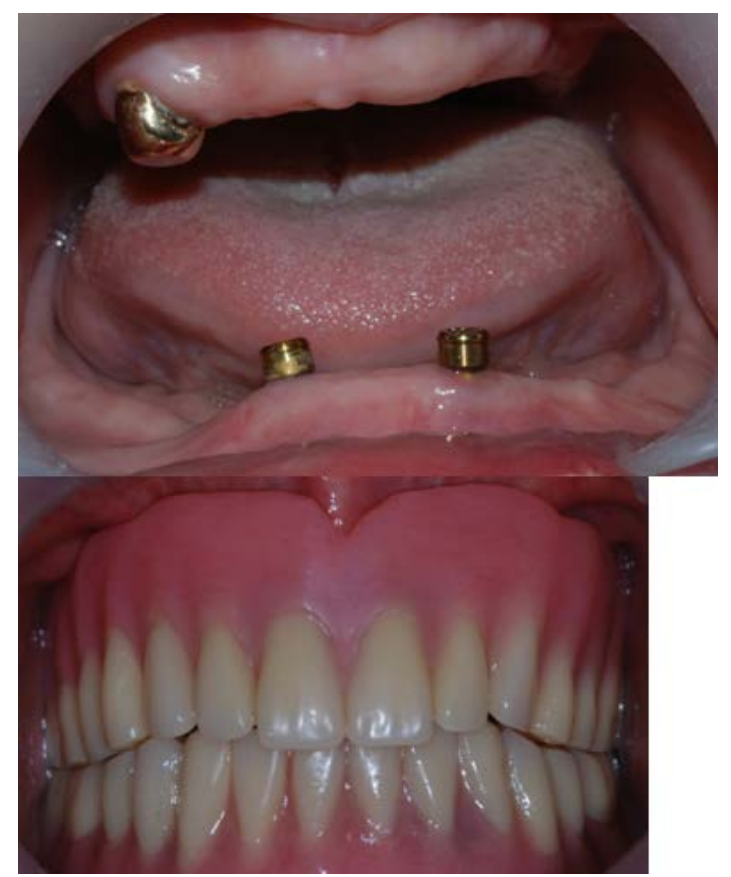

Figure 6. Magnetic attachment cemented at \#13 and Locator caps are selected for an abutment finish line of $2.5 \mathrm{~mm}$ above the gingival crest (Left), Try-on of prosthetics exhibits stable functional and esthetic results (Right)

Prosthetic space analysis revealed inter-ridge space as $16 \mathrm{~mm}$ in CR guided (Figure 4) and Magnetic device (Magfit, Aichi Steel Corporation, Japan) for maxilla and LODI system (ZEST Anchors, Escondido, CA, USA) for mandible were selected after spatial measurement of these attachment components. For dental implantation in mandible, anticoagulant therapy was stopped and value of 
INR assessed prior to implant surgery, followed by the installation of the implants $(2.4 \mathrm{~mm}, 13 \mathrm{~mm})$ (ZEST Anchors, Escondido, CA, USA) near the sites of \#32 and 42. Postoperative complications were minimal and healing was uneventful. Three months after implant placement, pending the osseointegration and gum healing, locator caps were screwed into place on the implants and were tightened to about $30 \mathrm{~N} / \mathrm{cm}$ (Figure 5). The height of cuff was selected for an abutment finish line of $2.5 \mathrm{~mm}$ above the gingival crest.

In maxilla, after root canal treatment of \#13, this tooth was used for a cap-type magnetic appliance on the occlusal surface of the root cap with ferrule effect for protection of abutments. Due to lower lateral forces transferred to abutments, the dowel post was limited less than $4 \mathrm{~mm}$ in height. Impression and laboratory processing were conducted under the protocol of complete denture fabrication. The stability, retention, lip support, esthetic, and denture were shown to be appropriately secured after 1 year follow check (Figure 6).

\section{Discussion}

Branemark protocol suggests, when compared to the fixed implant-supported restoration, the removable implant retained overdenture offers several advantages including enhanced access for oral hygiene, easy modification of the prosthesis base, and the provision of a labial flange to improve esthetics in situations of unfavorable jaw relationship [2]. In atrophied maxilla and mandible in this case, a lack of supporting bone can compromise the insertion of endosseous implants. The severely resorbed jaws can have some treatment options to enhance the prosthodontic results, iliac or chin bone grafting or distraction osteogenesis could be one of available alternatives to compensate those sequelae. However, patient in present case rejected major surgery demanding general anesthesia and hospitalization due to diabetes mellitus and prescription of anticoagulants (Warfarin) related with cardiovascular problem. He also had a fear of allograft even in minor surgical intervention too. Financial aspects were considered as well, general costs for full fixed implant supported restoration with bone grafting in bimaxillary edentulous state were about 3 to 4 times expensive those of implant overdenture therapy. Fortunately, patient has single sound canine to support attachment in maxilla. The single magnetic attachment provided the small denture bearing dimension with effective retention and stability. There are now overwhelming evidences that a two-implant overdenture is the minimum standard that is sufficient for most people [3], performance, patient satisfaction with minimum offered, cost and clinical time as well [4,5]. However, in this mandible anterior ridge, standard diameter $(4.0 \mathrm{~mm})$ implants could not be installed, it required at least $7 \mathrm{~mm}$ of bucco-lingual dimension to hold $2 \mathrm{~mm}$ of buccal bone from implant surface to avoid bone resorption and gingival shrinkage, Thus, using narrow-diameter implants (2.4 $\mathrm{mm}$ ) was able to present a solution of dimension obstacles without the need for additional grafting procedures under $5.5 \mathrm{~mm}$ available width measured at anterior ridge. Recently, mini dental implants are considered to be a good alternative to standard dental implants [6] and achieve a favorable primary stability for immediate loading placed into the inter-foraminal region [7]. Clinical stability (98.3\%) after 1 year of function [8] and a 5-year of benefit of treatment modality were demonstrated [9]. A resilient connector system, Locator selected can be applied in a limited inter-ridge space [10]. This abutment and attachment system's height is only 3.17 mm whereas bar attachment 12-14 mm, ball attachment 5$8 \mathrm{~mm}$. Locator attachments are also available in different vertical heights and abutment angle within 20 degree. Finally, it would be desirable for handicapped patient to rehabilitate chewing function with minimal prosthodontic and surgical approaches. Periodic dental and medical recall should be necessary and long-term clinical researches for narrow diameter implant overdenture are still required to maintain their longevity.

\section{References}

[1] van Waas MA. The influence of clinical variables on patients' satisfaction with complete dentures. J Prosthet Dent. 1990; 63: 307-10.

[2] Attard NJ, Laporte A, Locker D, Zarb GA. A prospective study on immediate loading of implants with mandibular overdentures: patient-mediated and economic outcomes. Int J Prosthodont. 2006; 19 (1): 67-73.

[3] Thomason JM, Feine J, Exley C, Moynihan P, Müller F, Naert I, Ellis JS, Barclay C, Butterworth C, Scott B, Lynch C, Stewardson D, Smith P, Welfare R, Hyde P, McAndrew R, Fenlon M, Barclay $\mathrm{S}$, Barker D. Mandibular two implant-supported overdentures as the first choice standard of care for edentulous patients-the York Consensus Statement. Br Dent J. 2009; 207 (4): 185-6.

[4] The McGill consensus statement on overdentures. European Journal of Prosthodontics and Restorative Dentistry 2002; 10: 956.

[5] Feine JS, Carlsson GE, Awad MA, Chehade A, Duncan WJ, Gizani S, Head T, Lund JP, MacEntee M, Mericske-Stern R, Mojon P, Morais J, Naert I, Payne AG, Penrod J, Stoker GT, Tawse-Smith A, Taylor TD, Thomason JM, Thomson WM, Wismeijer D. The McGill consensus statement on overdentures. Mandibular two-implant overdentures as first choice standard of care for edentulous patients. Int J Oral Maxillofac Implants. 2002; 17 (4): 601-2.

[6] Griffitts TM, Collins CP, Collins PC. Mini dental implants: an adjunct forretention, stability, and comfort for the edentulous patient. Oral Surg Oral Med Oral Pathol Oral Radiol. Endod. 2005; 100: e81-e84.

[7] Preoteasa E, Melescanu-Imre M, Preoteasa CT, Marin M, Lerner $\mathrm{H}$. Aspects of oral morphology as decision factors in mini-implant supported overdenture. Rom J Morphol Embryol. 2010; 51: 30914.

[8] Blaard A, Vanvr JB. Multi-clinic evaluation using mini-dental implants for long-term dental stabilization: A preliminary biometric evaluation. Compend Contin Educ Dent. 2005; 26: 8927.

[9] Mazor Z, Steigmann M, Leshem R, Peleg M. Mini-implants to reconstruct missing teeth in severe ridge deficiency and small interdental space: A 5-year case series. Implant Dent. 2004; 13: 336-41.

[10] Pasciuta M, Grossmann Y, Finger IM. A prosthetic solution to restoring the edentulous mandible with limited interarch space using an implant-tissue-supported overdenture: a clinical report. J Prosthet Dent. 2005; 93 (2): 116-20. 